\title{
Total parenteral nutrition in patients with advanced ovarian cancer
}

\author{
B. Madhok, S. Yeluri, A. Burton, K. Haigh and D. G. Jayne \\ Academic Unit of Colorectal Surgery and Clinical Nutrition, Clinical Sciences Building, The Leeds Teaching Hospitals \\ NHS Trust, St James University Hospital, Beckett Street, Leeds, West Yorkshire LS9 7TF, UK
}

The provision of total parenteral nutrition (TPN) to patients with disseminated ovarian cancer remains controversial due to unclear indications and unproven benefits ${ }^{(1,2)}$. The current study was undertaken to determine the pattern of prescribing in a large teaching hospital and to identify subgroups where TPN offered a potential benefit.

A prospectively maintained TPN database was used to identify sixty-five patients with disseminated ovarian carcinoma who received TPN between January 2002 and May 2008. Retrospective case note review was undertaken to retrieve data on patient demographics, disease stage, management and details of TPN prescribing.

The median age of the cohort was 67 (24-92) years. All patients had stage IIIc/IV ovarian carcinoma and had previously received chemotherapy and/or cytoreductive surgery. The indications for TPN were intestinal obstruction ( $n$ 23; 36\%), protracted post-operative ileus $(n 30 ; 46 \%)$, uncontrolled vomiting in the absence of bowel obstruction $(n 4 ; 6 \%)$ and enterocutaneous fistulas-short bowel syndrome $(n 8 ; 12 \%)$. Median survival from initiation of TPN was 4 (interquartile range (IQR) $1-12$ ) months. The median duration of TPN administration was 10 (5-19) d. Two subgroups were identified. Group 1 consisted of eighteen (28\%) patients who failed to benefit from TPN and were fed for a median of $5 \mathrm{~d}$. Ten $(56 \%)$ of these died of their disease and eight $(44 \%)$ had active treatment withdrawn whilst receiving TPN. The majority of these patients $(n 13 ; 72 \%)$ had disease-related terminal bowel obstruction. The median survival from starting TPN for these patients was 12 (IQR 6-28) d. Group 2 consisted of forty-seven (72\%) patients who derived a potential benefit from TPN and were fed for a median of $11 \mathrm{~d}$. Of these, twenty-five $(53 \%)$ had protracted post-operative ileus, four (9\%) had persistent vomiting unrelated to bowel obstruction, eight $(17 \%)$ had enterocutaneous fistulas-short bowel syndrome, ten $(21 \%)$ had disease-related bowel obstruction. The median survival from starting TPN in this group was 9 (IQR 3-14) months. In forty patients (85\%) enteral feeding was successfully recommenced whilst seven $(15 \%)$ required home TPN.

The decision to provide TPN to patients with disseminated ovarian cancer is often difficult and controversial. More than one-quarter of patients will derive no benefit and these include the majority of patients with disease-related terminal bowel obstruction. However, threequarters of patients may derive some benefit and these include patients with protracted post-operative ileus. Further studies are required to clarify the indications, cost-effectiveness and quality-of-life benefit of TPN in this group of patients.

1. Brard L, Weitzen S, Strubel-Lagan SL et al. (2006) Gynecol Oncol 103, 176-180.

2. Abu-Rustum NR, Barakat RR, Venkatraman E et al. (1997) Gynecol Oncol 64, 493-495. 